\title{
Boron Nitride Nanotubes Selectively Permeable to Cations or Anions
}

Tamsyn A. Hilder, ${ }^{\star}$ Daniel Gordon, and Shin-Ho Chung

\begin{abstract}
$\boldsymbol{B}_{\text {io }}$ ionic species. They maintain the resting membrane potential, generate propagated action potentials, and control a wide variety of cell functions. Here it is demonstrated theoretically that boron nitride nanotubes have the ability to carry out some of the important functions of biological ion channels. Boron nitride nanotubes with radii of 4.83 and $5.52 \mathrm{~A}$ embedded in a silicon nitride membrane are selectively permeable to cations and anions, respectively. They broadly mimic some of the permeation characteristics of gramicidin and chloride channels. Using distributional molecular dynamics, which is a combination of molecular and stochastic dynamics simulations, the properties of these engineered nanotubes are characterized, such as the free energy encountered by charged particles, the water-ion structure within the pore, and the current-voltage and current-concentration profiles. These engineered nanotubes have potential applications as sensitive biosensors, antibiotics, or filtration devices.
\end{abstract}

\author{
Keywords: \\ - engineered ion channels \\ - ion selectivity \\ - membranes \\ - molecular dynamics \\ - nanotubes
}

\section{Introduction}

The cell membrane presents a large electrostatic barrier that is impermeable to charged particles such as sodium, chloride, and potassium ions. Biological ion channels, or bionanotubes, regulate the movement of ions across the cell membrane by providing a water-filled pore through which ions can pass. They are found in all living cells, from plants and bacteria to humans. By allowing specific ionic species to move across the cell membrane, bio-nanotubes regulate all electrical activities in the nervous system, including communication between cells and the influence of hormones and drugs on cell function. ${ }^{[1]}$

Several attempts have been made in the past to engineer nanotubes that can carry out some of the functions performed by biological channels. Carbon nanotubes embedded into a lipid bilayer ${ }^{[2,3]}$ and in artificial membranes ${ }^{[4-8]}$ pass water molecules across with a conduction rate comparable to that of

[*] Dr. T. A. Hilder, Dr. D. Gordon, Prof. S.-H. Chung Computational Biophysics Group, Research School of Biology Australian National University, ACT 2601 (Australia)

E-mail: tamsyn.hilder@anu.edu.au

DOI: $10.1002 /$ smll.200901229 the biological water channel, aquaporin-1. In some cases this can occur while effectively rejecting charged particles. ${ }^{[8-10]}$ Although work on carbon nanotubes has predominantly focused on the conduction of water molecules, recently their ion selectivity has been investigated. ${ }^{[11-14]}$ When a rim of partially charged atoms are placed near the entrance, ions surmount the energy barrier near the open-ended entrance and enter the interior of a carbon nanotube. ${ }^{[11-13]}$ Joseph et al. ${ }^{[11]}$ have shown that ion occupancy is significantly increased. Similarly, Majumder et al. ${ }^{[13]}$ have shown that placing negatively charged functional groups at the carbon nanotube tips significantly increases the flux of positive ions, but this effect is reduced at higher ionic concentrations. Using molecular dynamics simulations, Park et al. ${ }^{[12]}$ were able to separate potassium and chloride ions using a $\mathrm{Y}$-junction carbon nanotube constructed from an $(8,8)$ main branch nanotube connected to two smaller $(5,5)$ and $(6,6)$ branches. The negatively charged $(5,5)$ nanotube was found to conduct potassium ions and the positively charged $(6,6)$ nanotube conducted chloride ions, but at certain charge densities potassium ions were shown to enter the $(6,6)$ nanotube. The nanotube diameter can also be used to select ions by molecular sieving. ${ }^{[14]}$

Single-walled nanotubes may be manufactured from various materials other than carbon, such as boron nitride. ${ }^{[15]}$ 
In comparison to carbon nanotubes, boron nitride nanotubes exhibit improved properties, such as superior biocompatibility. ${ }^{[16]}$ Unlike carbon nanotubes, boron nitride nanotubes are inherently non-cytotoxic. ${ }^{[17]}$ Moreover, the atoms in boron nitride nanotubes are polarized, with boron atoms becoming slightly positive with respect to the nitrogen atoms. ${ }^{[18]}$ The polarization of the atoms lining the pore causes a boron nitride nanotube to be more permeable to water molecules compared to carbon nanotubes of similar radius and length. ${ }^{[19-21]}$ This difference in electronegativity has a pronounced influence on the geometry of water molecules within the pore, and by increasing the nanotube radius the configuration of water molecules, constrained by the polarized side-wall atoms, undergoes systematic changes.

Here we show for the first time that boron nitride nanotubes with radii of 4.83 and $5.52 \AA$ embedded in a silicon nitride membrane broadly mimic the biological gramicidin and chloride channels by being exquisitely selective to cations and anions, respectively. Unlike in biological systems, where cation-anion selectivity is determined by charged residues lining the pore, the selectivity in boron nitride nanotubes is conferred by the intricate interactions between the polar water molecules, ions, charge differences between boron and nitrogen atoms forming the pore, and permanent dipoles surrounding the nanotube surface. Using the technique of distributional molecular dynamics (DMD),${ }^{[22]}$ which combines classical molecular dynamics (MD) with stochastic dynamics, we characterize the boron nitride nanotubes that are selectively permeable to cations and anions and provide the mechanisms underlying their selectivity.

\section{Results and Discussion}

We consider open-ended, single-walled $(7,7)$ and $(8,8)$ boron nitride nanotubes with a length of approximately 24 $26 \AA$, and radii of 4.83 and $5.52 \AA$, respectively, fixed in a silicon nitride membrane ${ }^{[23]}$ surrounded by a reservoir containing a $500 \mathrm{~mm}$ ionic solution such as $\mathrm{NaCl}$, as illustrated in Figure 1A. The boron nitride nanotubes are constructed from a hexagonal array $^{[24]}$ of alternating boron and nitrogen atoms rolled up to form a tubular structure. ${ }^{[25]}$ In MD simulations of the $(7,7)$ and $(8,8)$ nanotubes, water molecules are rapidly drawn into the interior of the pore in the absence of any applied pressure. ${ }^{[10]}$ Water encapsulated inside the $(7,7)$ and $(8,8)$ pores is observed to form ordered spiral and helical structures, respectively (Figure 1B). Such highly ordered, ice-like water structures have been observed previously in narrow confines of carbon nanotubes. ${ }^{[26,27]}$ Under the influence of an applied potential, a cation or chloride ion enters the $(7,7)$ or $(8,8)$ pore, respectively, and replaces one of the water molecules in the chain, as shown in Figure 1B(I) and Figure 1B(III) respectively.

Single-walled, $(7,7)$ boron nitride nanotubes with a radius of $4.83 \AA$ and a length of approximately $26 \AA$ broadly mimic the biological gramicidin channel by being selectively permeable to cations, and rejecting chloride. The profile of free energy, shown in Figure 2A, reveals that monovalent cations $\left(\mathrm{Na}^{+}, \mathrm{K}^{+}\right.$, and $\left.\mathrm{Li}^{+}\right)$encounter an energy well as they traverse the conduit.
A)

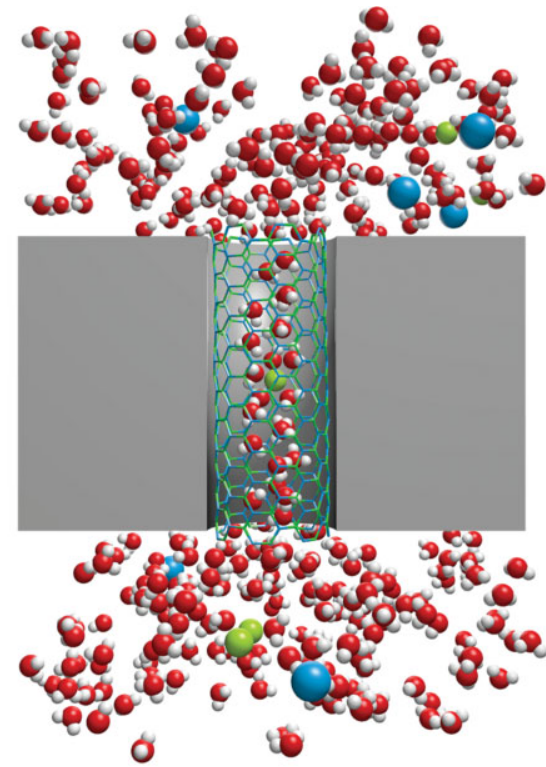

B)
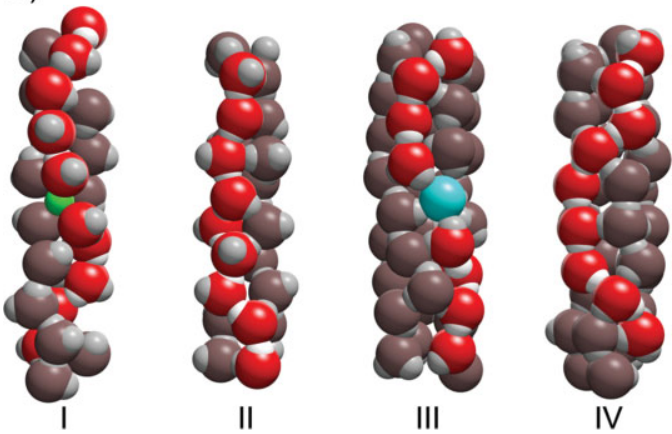

Figure 1. Visualization of water and ions inside a boron nitride nanotubeembedded silicon nitride membrane. A) Boron nitride nanotube shown inside a silicon nitride matrix (grey) between two reservoirs containing water and ions. Note that for clarity not all water molecules and ions are shown. B) Ordered spiral and helical water structures within the $(7,7)$ and $(8,8)$ boron nitride nanotubes, respectively. I) With and II) without a sodium ion in the $(7,7)$ nanotube. III) With and IV) without a chloride ion in the $(8,8)$ nanotube.

In contrast, there exists an extremely large energy barrier for chloride $\left(\mathrm{Cl}^{-}\right)$to overcome.

The current-voltage and current-concentration profiles constructed using DMD are displayed in Figure 2B and C. The current increases linearly with an applied potential and with an increasing ionic concentration. The conductance, derived by fitting a linear regression through the data points, is $173.2 \pm 7.5 \mathrm{pS}$ for potassium, $129 \pm 4.8 \mathrm{pS}$ for sodium, and $81 \pm 9.2 \mathrm{pS}$ for lithium. All investigated cations exhibit a conductance approximately 5-9 times larger than gramicidin, which has a conductance of approximately $15-20 \mathrm{pS}$. That the current-concentration relationship is linear, rather than that of the Michaelis-Menten form, indicates that the monovalent cations traversing the pore do not encounter any energy barrier, and the rate limiting step in the conduction process is the time it takes for the ions to enter the pore. ${ }^{[28]}$ Once in the pore, the ion rapidly traverses the channel a single ion at a time. 

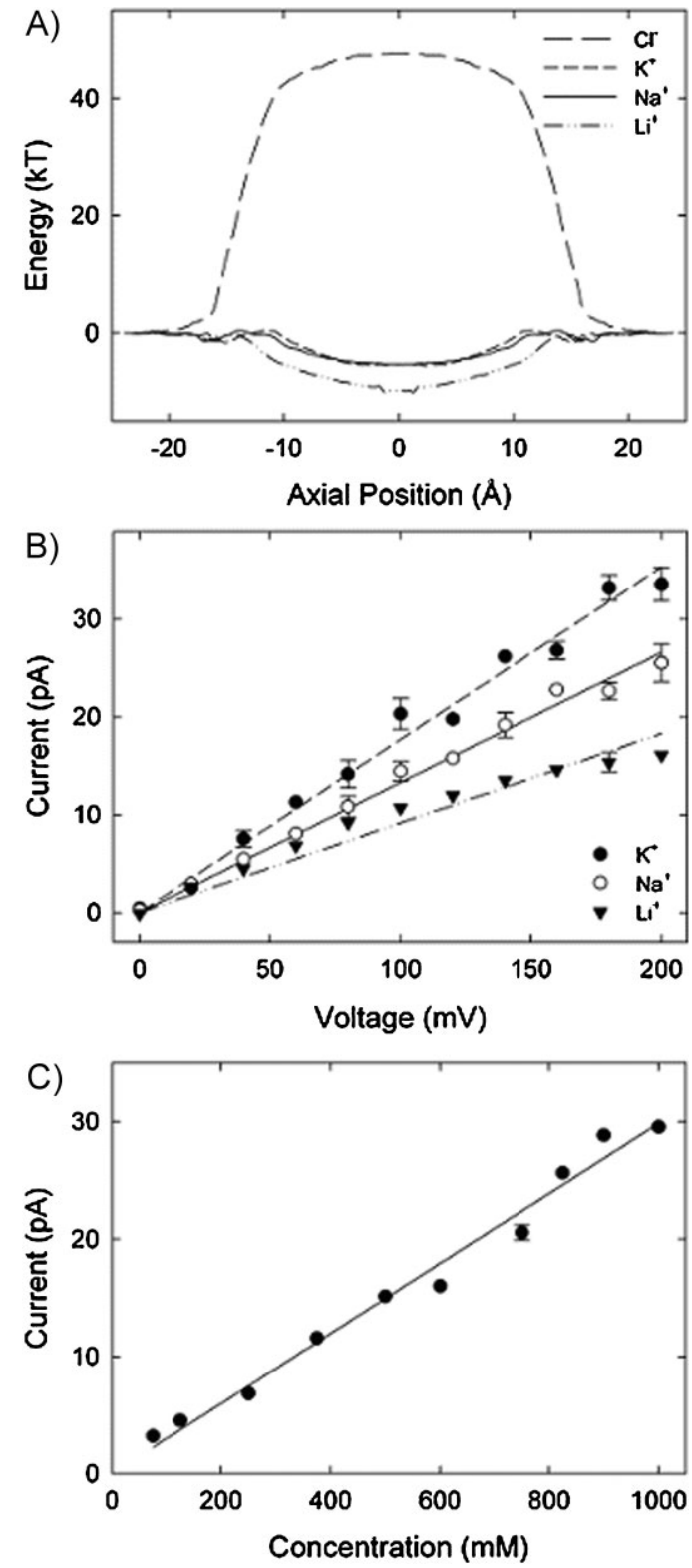

Figure 2. Cation-selective $(7,7)$ boron nitride nanotube. A) Free-energy profile for sodium $\left(\mathrm{Na}^{+}\right)$, potassium $\left(\mathrm{K}^{+}\right)$, lithium $\left(\mathrm{Li}^{+}\right)$, and chloride $\left(\mathrm{Cl}^{-}\right)$. B) Current-voltage curve for the monovalent cations sodium, potassium and lithium. C) Current-concentration curve for sodium. Note that error bars represent one standard error of the mean and error bars smaller than the data points are not shown.

As we have noted above, this cation-selective behavior is exhibited in nature through the gramicidin channel. Gramicidin A, a small peptide-based antibiotic, was one of the first antibiotics isolated and used in clinical practice. ${ }^{[29]}$ It forms a cylindrical channel across the bacteria cell membrane with a radius of approximately $2 \AA$, which selectively conducts monovalent cations. ${ }^{[30]}$ Once formed, these cations rapidly move across the cell and destroy the membrane potential, thus killing the bacteria cell. The interior surface of gramicidin is lined with polar groups that aid the conduction of ions and water molecules, and the pore size is such that the ions and water molecules are restricted to move in a highly correlated singlefile chain. ${ }^{[29]}$ In the $(7,7)$ boron nitride nanotube there is a unique interaction between the boron and nitrogen atoms with the uniform permanent dipoles of the silicon nitride membrane, and the ions and water molecules move in a highly ordered spiral chain (Figure $1 \mathrm{~B}(\mathrm{I})$ and Figure $1 \mathrm{~B}(\mathrm{II})$ ). The ability to mimic gramicidin's physiological attributes could lead to applications such as new antibiotic treatments, biosensors, and cancer treatment.

In contrast, we find that the single-walled, $(8,8)$ boron nitride nanotubes with a radius of $5.52 \AA$ and a length of approximately $24 \AA$ are selectively permeable to chloride ions and reject all cationic species, thus broadly mimicking biological chloride channels (e.g., $\mathrm{ClC}-1$ ). The profile of free energy, shown in Figure $3 \mathrm{~A}$, reveals that a chloride ion encounters an energy well as it traverses the conduit. In contrast, there exists a large energy barrier for both sodium and cesium $\left(\mathrm{Na}^{+}\right.$and $\left.\mathrm{Cs}^{+}\right)$to overcome.

The current-voltage and current-concentration profiles are displayed in Figure $3 \mathrm{~B}$ and $\mathrm{C}$, respectively. The current increases linearly with an applied potential and with an increasing ionic concentration. The chloride conductance, derived by fitting a linear regression through the data points, is $14.7 \pm 0.8 \mathrm{pS}$. The conductance of the engineered channel is approximately equal to that of the $\mathrm{ClC}-1$ channel, which is $8.5 \mathrm{pS}$ with symmetrical solutions of $150 \mathrm{~mm}$ and $14 \mathrm{pS}$ with $500 \mathrm{~mm},{ }^{[31]}$ but a third that of the $\mathrm{GABA}_{\mathrm{A}}$ receptor. ${ }^{[32]}$ As with the $(7,7)$ boron nitride nanotube, the current-concentration relationship is linear, rather than that of the Michaelis-Menten form (Figure 3C). Similarly, the pore attracts a single ion that once in the pore rapidly traverses the channel.

Anion-selective biological channels are involved in a diverse range of physiological functions, such as the control of cellular excitability, acidification of intracellular vesicles, and regulation of cell volumes. Although both $(8,8)$ boron nitride nanotubes and eukaryotic $\mathrm{ClC}$ channels are exquisitely selective to anions, their mechanisms for achieving this high selectivity differ. In $\mathrm{ClC}-1$, the conduction path is lined with 4 acidic and 10 basic residues. ${ }^{[31]}$ The net positive charges on the protein wall forming the conduit create a deep energy well of $\approx 47 \mathrm{kT}$ for an anion, which attracts on average 2.3 chloride ions. A third ion entering the pore, driven by an applied potential, disrupts the stable equilibrium established by the resident ions, and the outer ion is expelled from the pore through a Coulomb repulsion. In the engineered channel, it is a combination of the hydration force and the unique interaction between the boron and nitrogen atoms of the nanotube with the permanent dipole of the silicon nitride membrane that are solely responsible for selectivity of anions over cations, and the energy well attracts a single $\mathrm{Cl}^{-}$, which is expelled from the pore through Coulomb repulsion once a second ion enters.

We find that in both boron nitride nanotubes ion selectivity is a consequence of the unique water structure within the nanotube, the nanotube radius, the partial charge on the boron and nitrogen atoms of the nanotube, and the permanent dipoles existing in the silicon nitride membrane. As the cation or anion enters the narrow nanotube pore it must lose part of its solvation shell. In bulk water ions are usually bound by between four and six water molecules, and these ions are called solvated 

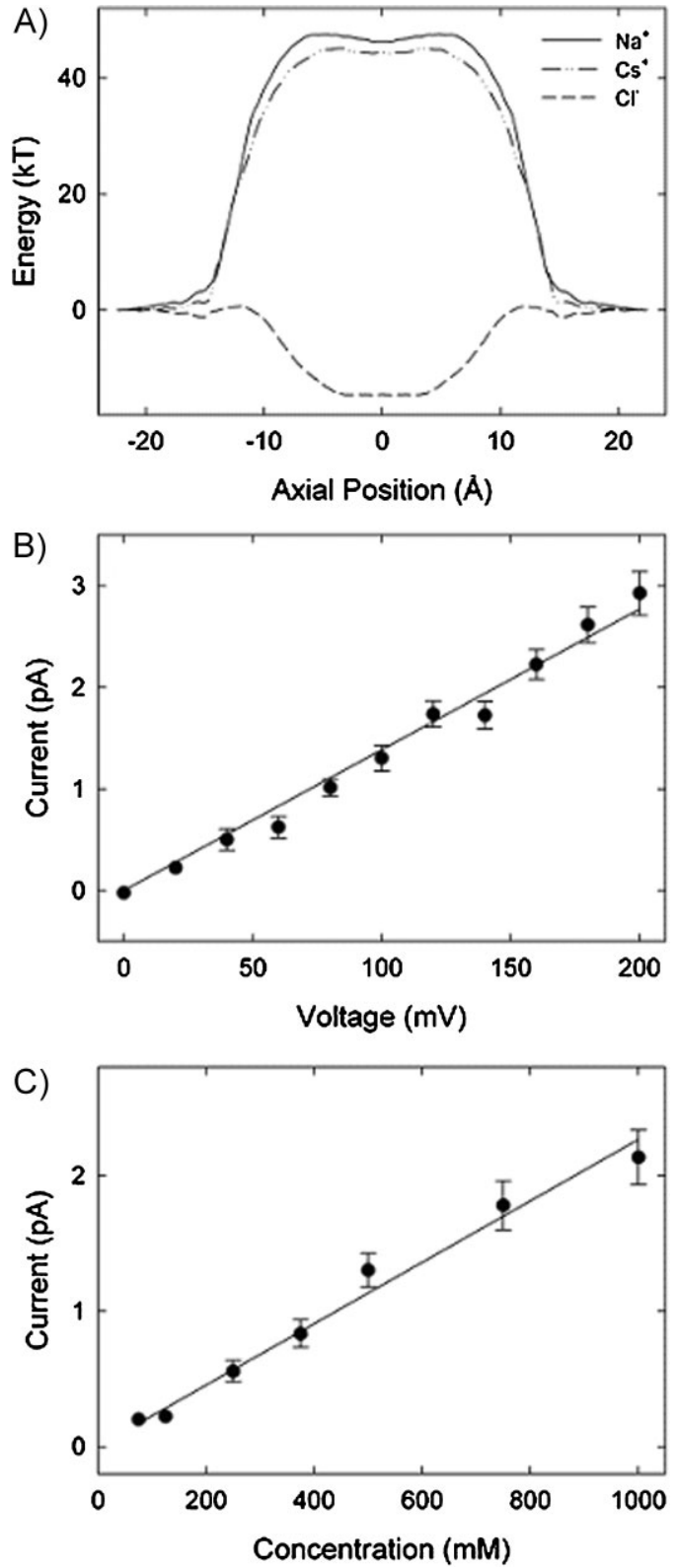

Figure 3. Anion-selective $(8,8)$ boron nitride nanotube. A) Free-energy profile for sodium $\left(\mathrm{Na}^{+}\right)$, cesium $\left(\mathrm{Cs}^{+}\right)$, and chloride $\left(\mathrm{Cl}^{-}\right)$. B) Currentvoltage curve for chloride ions. C) Current-concentration curve for chloride ions. Note that error bars represent one standard error of the mean and error bars smaller than the data points are not shown.

or hydrated ions. There is a large energetic cost, dehydration energy, associated with removing water molecules from this tightly bound hydration shell. The unique water structure within the nanotube and partial charges on the boron and nitrogen atoms provide a favorable environment to restore part of the energy lost from dehydration.

It is not known whether the silicon nitride membrane that forms around the boron nitride nanotube will be an ordered crystal lattice, as assumed by previous molecular dynamics studies, ${ }^{[33,34]}$ or an amorphous structure. Nevertheless, it is convenient to use the silicon nitride matrix as a means of applying a uniform dipole at the pore surface. The polarity of the permanent dipole created by the matrix reverses depending on the size of the embedded pore. According to ab initio density functional theory typically the nitrogen atoms of the boron nitride nanotube shift outwards ${ }^{[18]}$ due to the electronic structure by $0.061 \AA$. We confirm these results using both Gaussian03 and CPMD (unpublished). With a $(7,7)$ boron nitride nanotube embedded in a silicon nitride membrane, nitrogen atoms in the matrix surround the outer surface of the pore, thus causing the boron atoms of the tube to shift slightly outward relative to the nitrogen atoms of the tube by $0.0071 \AA$. Conversely, with an $(8,8)$ nanotube, silicon atoms surround the surface of the pore, and the nitrogen atoms of the tube move outward relative to the boron atoms by $0.0131 \AA$. Small shifts in the relative positions of the boron and nitrogen atoms partially account for the anion/cation selectivity observed in our study. The effect of permanent dipoles provided by the crystalline silicon nitride membrane may be achieved by suitable functionalization of the boron nitride nanotubes such as by polymer wrapping ${ }^{[35]}$ with oriented permanent dipoles, or covalent functionalization. ${ }^{[36]}$

Engineered, cation-selective nanotubes firmly embedded across the semipermeable membrane will disrupt the electrochemical gradients that maintain the passive resting membrane potential. The concentration of potassium ions inside of the cell is high relative to the outside $(150 \mathrm{~mm}$ vs. $5 \mathrm{~mm})$. This concentration gradient across the membrane gives rise to a resting potential, making the interior of the cell negative with respect to the outside. The sodium concentration in the interior of a living cell is very low, whereas that in the external medium is high. Thus, when cation-selective nanotubes are inserted across the membrane, sodium ions in the extracellular medium will rush into the cell interior, driven by the concentration as well as electrical gradients. Under these conditions, the resting membrane potential will be destroyed, thus making the cell die. This disruption of the resting potential is the basis for the potent antimicrobial action of the gramicidin pore. Similarly, the insertion of chloride nanotubes across the cell membrane will disrupt the dynamic equilibrium of ionic concentrations between the interior of the cell from the extracellular space. The membrane will become leaky, as a high external chloride concentration will cause a continuous influx of anions from outside to inside. The membrane potential of a cell under this condition will be permanently clamped at the equilibrium potential of chloride (about $-70 \mathrm{mV}$ in mammalian neurons), thus causing the cell to be incapable of generating propagated action potentials.

\section{Conclusions}

We show that boron nitride nanotubes embedded in a silicon nitride membrane ${ }^{[22]}$ can be engineered such that they are either cation- or anion-selective by varying the nanotube diameter. This selectivity is dependent on the pore size, the uniform permanent dipole surrounding the nanotube surface, and the unique structure of water within the nanotube. Our study demonstrates that there is a real possibility of fabricating 
and functionalizing boron nitride nanotubes that are selectively permeable to cations or anions. Mimicking biological ion channels such as gramicidin and $\mathrm{ClC}$ suggests the prospect of creating many novel medical devices. Several inherited neurological, muscular, and renal disorders arise from malfunctioning of ion channels such as epilepsy, cystic fibrosis, and diabetes. It is only a matter of time until engineered channels, making use of the advanced technology in nanosciences, will be grafted onto biological membranes, leading to drugs specifically targeted at ion channels. These specifically, synthetic nanotubes can be specifically targeted to certain cell types, making them unviable by causing their cell membrane to become leaky, thus leading to a host of pharmaceutical products to assist in treatments such as antibacterial, cancer, and cystic fibrosis. The main features of synthetic nanotubes that suggest their extraordinary potential as artificial ion channels are i) the ability to precisely control their length to target cells with a specific cell thickness, for example, bacteria cell membranes are thinner than human cell membranes, ii) a drastically enhanced ionic transport compared to biological ion channels, and iii) the ability to modify their surface chemistry to target various cells, from bacteria to cancer. In addition, these boron nitride embedded silicon nitride membranes may be used directly in engineered membrane applications such as sensitive chemical and biological biosensors and as desalination devices.

\section{Experimental Section}

Boron nitride nanotube construction: Single-walled, boron nitride nanotubes were constructed from a hexagonal array ${ }^{[24]}$ of alternating boron and nitrogen atoms rolled up to form a tubular structure $^{[25]}$ similar to the carbon nanotube. The boron nitride nanotube is defined by the chiral vector, $C=n a_{1}+m a_{2}=(n, m)$, where $a_{1}$ and $a_{2}$ represent the unit vectors of the hexagonal lattice. ${ }^{[37]}$ For simplicity, we consider only armchair tubes, which are defined by $C=(n, n)$. Specifically, we investigated the $(7,7)$ and $(8,8)$ boron nitride nanotubes embedded in a silicon nitride membrane. ${ }^{[23]}$ The boron nitride nanotubes were constructed using the basic relations governing the fundamental parameters of a carbon nanotube $e^{[37]}$ and a boron-nitrogen bond distance ${ }^{[20]}$ of $1.446 \AA$. The Lennard-Jones constants for boron and nitrogen atoms were obtained from Won and Aluru. ${ }^{[19]}$ Each boron and nitrogen atom forming the boron nitride nanotube acquires a partial charge, and these atoms become further polarized in the presence of water. It is uncertain which values for the partial charge are most suitable to represent the electrostatic interaction in MD simulations. Therefore, for consistency, we use the partial charges determined using density functional theory by Won and Aluru, ${ }^{[20]}$ and in particular we use the values determined without water $( \pm 0.4 e)$ for all tubes. Investigation into suitable partial charge parameters for MD simulations is currently being explored by the authors (unpublished).

Molecular dynamics simulations: Molecular dynamics simulations were performed using $\mathrm{NAMD}^{[38]}$ and visualized using VMD. ${ }^{[39]}$ The MD domain consisted of a boron nitride nanotube, a silicon nitride matrix, water, and ions (either potassium, sodium, or lithium and chloride). The $(7,7)$ and $(8,8)$ boron nitride nanotubes were fixed in a silicon nitride membrane ${ }^{[23]}$ surrounded by a reservoir containing a $500 \mathrm{~mm}$ ionic solution such as $\mathrm{NaCl}$, as illustrated in Figure 1A. The silicon nitride matrix was constructed using methodology outlined in the bionanotechnology VMD and NAMD online tutorial. ${ }^{[40]}$ The simulation box for all runs was approximately $4.5 \times 4 \times 6.4 \mathrm{~nm}^{3}$. The system was replicated periodically in all three dimensions. The simulation box contained approximately 1200 water molecules, and 6-7 ion pairs for an ion concentration of $0.5 \mathrm{M}$. CHARMM $27^{[41]}$ force-field parameters and the TIP3 water model were used for all simulations. The system was equilibrated for $1 \mathrm{~ns}$ to a constant temperature of $310 \mathrm{~K}$ and constant pressure of 1 bar. The potential of mean force (PMF) of the specific ion moving through the investigated nanotubes was calculated using umbrella sampling. For all runs the silicon nitride matrix and the boron nitride nanotube were restrained with a harmonic constraint of 1.0 and $0.5 \mathrm{kcal} \mathrm{mol}^{-1} \AA^{-1}$, respectively, with the former chosen to fit the dielectric constant of silicon nitride. ${ }^{[40]}$ The ion was moved through positions, $z_{0}$ from 0 to 23 in $0.5 \AA$ increments and held in position using a harmonic constraint of $0.5 \mathrm{kcal} \mathrm{mol}^{-1} \AA^{-1}$. Each window was run for a total of $1 \mathrm{~ns}$. Runs were then analyzed using the weighted histogram analysis method. ${ }^{[42]}$

Distributional molecular dynamics: The free energy profile determined from MD simulations and the distribution of frictional forces that are measured over discrete segments of the channel were incorporated into the stochastic dynamics simulations based on the Langevin equation. This technique, which combines molecular and stochastic dynamics, termed DMD, ${ }^{[22]}$ enables the permeation of ions across these channels to be determined at much longer time scales than possible using MD simulations (the order of many hundreds of nanoseconds to a few microseconds). Using this method we were able to generate current-voltage and current-concentration curves and determine ion binding sites within the nanotube. The theoretical basis for this procedure and a detailed test using gramicidin-A is given by Gordon et al. ${ }^{[22]}$ Relaxation time constants $\gamma^{-1}$ were obtained from Hille ${ }^{[1]}$ and using the Einstein relation $D=k T /(m \gamma)$, where $D$ is the diffusion coefficient, $k$ is Boltzmann's constant, $T$ is the temperature, and $m$ is mass. All current-voltage curves were generated at an ionic concentration of $0.5 \mathrm{M}$. Current was calculated using the relationship $I=q n / \Delta t$, where $n$ is the average number of ions that cross the membrane, $q$ is the charge of the ion, and $\Delta t$ is the simulation time of one run. The conductance is then given by the slope of the linear regression curve fitted through all data points. Currentconcentration curves, which were calculated for sodium in the $(7,7)$ nanotube and chloride in the $(8,8)$ nanotube, were generated at a voltage of $100 \mathrm{mV}$.

\section{Acknowledgements}

This work was supported by the National Health and Medical Research Council. We acknowledge the use of the Australian National University Supercomputer Facility. We thank Ganesh 
Venkateshwara, Rui Yang, Alistair Rendell, Andrey Bliznyuk, Silvie Ngo, and Rhys Hawkins.

[1] B. Hille, Ionic channels of excitable membranes, 2nd ed. Sinauer Associates, Inc, Sunderland, MA 1992.

[2] C. F. Lopez, S. O. Nielsen, P. B. Moore, M. L. Klein, Proc. Natl. Acad. Sci. U. S. A. 2004, 101, 4431-4434.

[3] J.-A. Garate, N. J. English, J. M. D. MacElroy, Mol. Simul. 2009, 35, 2-12.

[4] G. Hummer, J. C. Rasaiah, J. P. Noworyta, Nature 2001, 414, 188190.

[5] A. Waghe, J. C. Rasaiah, G. Hummer, J. Chem. Phys. 2002, 117, 10789-10795.

[6] M. Majumder, N. Chopra, R. Andrews, B. J. Hinds, Nature 2005, 438, 44.

[7] A. Kalra, S. Garde, G. Hummer, Proc. Natl. Acad. Sci. U. S. A. 2003, 100, 10175-10180.

[8] B. Corry, J. Phys. Chem. B 2008, 112, 1427-1434.

[9] F. Fornasiero, H. G. Park, J. K. Holt, M. Stadermann, C. P. Grigoropoulos, A. Noy, O. Bakajin, Proc. Natl. Acad. Sci. U. S. A. 2008 , 105, 17250-17255.

[10] T. A. Hilder, D. Gordon, S. H. Chung, Small 2009, in press DOI: 10.1002/smll.200900349.

[11] S. Joseph, R. J. Mashi, E. Jakobsson, N. R. Aluru, Nano Lett. 2003, 3, 1399-1403.

[12] J. H. Park, S. B. Sinnott, N. R. Aluru, Nanotechnology 2006, 17, 895-900.

[13] M. Majumder, N. Chopra, B. J. Hinds, J. Am. Chem. Soc. 2005, 127, 9062-9070.

[14] H. Liu, C. J. Jameson, S. Murad, Mol. Simul. 2008, 34, 169-175.

[15] P. Fortina, L. J. Kricka, S. Surrey, P. Grodzinski, Trends Biotechnol. 2005, 23, 168-173.

[16] V. L. Solozhenko, A. G. Lazarenko, J.-P. Petitet, A. V. Kanaev, J. Phys. Chem. Solids 2001, 62, 1331-1334.

[17] X. Chen, P. Wu, M. Rousseas, D. Okawa, Z. Gartner, A. Zettl, C. R. Bertozzi, J. Am. Chem. Soc. 2009, 131, 890-891.

[18] N. Park, J. Cho, H. Nakamura, J. Phys. Soc. Jpn. 2004, 73, 24692472.

[19] C. Y. Won, N. R. Aluru, J. Am. Chem. Soc. 2007, 129, 2748-2749.

[20] C. Y. Won, N. R. Aluru, J. Phys. Chem. C 2008, 112, 1812-1818.

[21] M. E. Suk, A. V. Raghunathan, N. R. Aluru, Appl. Phys. Lett. 2008, 92, 133120.

[22] D. Gordon, V. Krishnamurthy, S. H. Chung, J. Chem. Phys. 2009, in press.

[23] J. K. Holt, A. Noy, T. Huser, D. Eaglesham, O. Bakajin, Nano Lett. 2004, 4, 2245-2250.

[24] P. Widmayer, H.-G. Boyen, P. Ziemann, P. Reinke, P. Oelhafen, Phys. Rev. B: Condens. Matter Mater. Phys. 1999, 59, 5233-5241.

[25] M. Ishigami, S. Aloni, A. Zettl, AIP Conf. Proc. 2003, 696, 94-99.

[26] K. Koga, R. D. Parra, H. Tanaka, X. C. Zeng, J. Chem. Phys. 2000, 113, 5037-5040.
[27] K. Koga, R. D. Parra, H. Tanaka, X. C. Zeng, Nature 2001, 412, 802805.

[28] S. H. Chung, T. W. Allen, M. Hoyles, S. Kuyucak, Biophys. J. 1999, 77, 2517-2533.

[29] O. S. Andersen, R. E. Koeppe II, B. Roux, in Biological Membrane Ion Channels: Dynamics, Structure, and Application (Eds: S. H. Chung, O. S. Andersen, V. Krishnamurthy ), Springer, New York 2007, pp. 33-80.

[30] S. Kuyucak, O. S. Andersen, S. H. Chung, Rep. Prog. Phys. 2001, 64, 1427-1472.

[31] B. Corry, M. O'Mara, S. H. Chung, Biophys. J. 2004, 86, 846-860.

[32] M. L. O’Mara, B. Cromer, M. W. Parker, S. H. Chung, Biophys. J. 2005, 88, 3286-3299.

[33] J. B. Heng, A. Aksimentiev, C. Ho, P. Marks, Y. V. Grinkova, S. Sligar, K. Schulten, G. Timp, Nano Lett. 2005, 5, 1883-1888.

[34] A. Aksimentiev, J. B. Heng, G. Timp, K. Schulten, Biophys. J. 2004, 87, 2086-2097.

[35] C. Zhi, Y. Bando, C. Tang, R. Xie, T. Sekiguchi, D. Golberg, J. Am. Chem. Soc. 2005, 127, 15996-15997.

[36] X. Wu, W. An, X. C. Zeng, J. Am. Chem. Soc. 2006, 128, 1200112006

[37] M. S. Dresselhaus, G. Dresselhaus, R. Saito, Carbon 1995, 33, 883-891.

[38] L. Kale, R. Skeel, M. Bhandarkar, R. Brunner, A. Gursoy, N. Krawetz, J. Phillips, A. Shinozaki, K. Varadarajan, K. Schulten, J. Comput. Phys. 1999, 151, 283-312; NAMD, available from http:// www.ks.uiuc.edu/Research/namd/ (accessed August 2008).

[39] W. Humphrey, A. Dalke, K. Schulten, J. Molec. Graphics 1996, 14, 33-38; VMD, available from http://www.ks.uiuc.edu/Research/ vmd/ (accessed August 2008).

[40] University of Illinois, Urbana-Champaign, Beckman Institute for Advanced Science and Technology, Theoretical and Compuational Biophysics Group. Bionanotechnology tutorial, http:// www.ks.uiuc.edu/Training/Tutorials/science/bionano/bionanotutorial.pdf (accessed July 2008)

[41] a) B. R Brooks, R. E. Bruccoleri, B. D. Olafson, D. J. States, S. Swaminathan, M. Karplus, J. Comput. Chem. 1983, 4, 187217. b) A. D. MacKerell Jr., B. Brooks, C. L. Brooks III, L. Nilsson, B. Roux, Y. Won, M. Karplus, in: The Encyclopedia of Computational Chemistry, Vol. 1(Eds: P. V. R. Schleyer, P. R. Schreiner, N. L. Allinger, T. Clark, J. Gasteiger, P. Kollman, H. F. III ), John Wiley \& Sons, Chichester 1998, pp. 271-277.

[42] a) Kumar, S. Schaefer J. M. Rosenberg, D. Bouzida, R. H. Swendsen, P. A. Kollman, J. Comput. Chem. 1995, 16, 1339-1350. b) A. Grossfield, An implementation of WHAM: the weighted histogram analysis method, http://membrane.urmc.rochester. edu/Software/WHAM/WHAM.html (accessed August 2008).

Received: July 14, 2009

Revised: August 7, 2009

Published online: September 30, 2009 